\title{
Effect of PEG-PDLLA polymeric nanovesicles loaded with doxorubicin and hematoporphyrin monomethyl ether on human hepatocellular carcinoma HepG2 cells in vitro
}

\author{
This article was published in the following Dove Press journal: \\ International Journal of Nanomedicine \\ 29 November 2013 \\ Number of times this article has been viewed
}

\author{
Guang-Hua Xiang ${ }^{1,2, *}$ \\ Guo-Bin Hong ${ }^{2,3, *}$ \\ Yong Wang ${ }^{2}$ \\ Du Cheng ${ }^{2}$ \\ Jing-Xing Zhou' \\ Xin-Tao Shuai ${ }^{2}$
}

'Department of Radiology, Sun Yat-Sen Memorial Hospital, Sun Yat-Sen University, Guangzhou, People's Republic of China; ${ }^{2}$ PCFM Laboratory of Ministry of Education, School of Chemistry and Chemical Engineering, Sun Yat-Sen University, Guangzhou, People's Republic of China; ${ }^{3}$ Department of Radiology, Fifth Affiliated Hospital, Sun Yat-Sen University, Zhuhai, People's Republic of China

*These two authors contributed equally to this work
Correspondence: Du Cheng PCFM Laboratory of Ministry of Education, School of Chemistry and Chemical, Engineering, Sun Yat-Sen University, Guangzhou 510275, People's Republic of China Email chengdu@mail.sysu.edu.cn

Jing-Xing Zhou

Department of Radiology, Sun YatSen Memorial Hospital, Sun Yat-Sen University, Guangzhou 5I0I20,

People's Republic of China

Email gdzhoujx@163.com
Objective: To evaluate the cytotoxicity of poly(ethylene glycol)-block-poly(D,L-lactic acid) (PEG-PDLLA) nanovesicles loaded with doxorubicin (DOX) and the photosensitizer hematoporphyrin monomethyl ether (HMME) on human hepatocellular carcinoma HepG2 cells and to investigate potential apoptotic mechanisms.

Methods: PEG-PDLLA nanovesicles were simultaneously loaded with DOX and HMME (PEG-PDLLA-DOX-HMME), and PEG-PDLLA nanovesicles were loaded with DOX (PEGPDLLA-DOX), HMME (PEG-PDLLA-HMME), or the PEG-PDLLA nanovesicle alone as controls. The cytotoxicity of PEG-PDLLA-DOX-HMME, PEG-PDLLA-DOX, PEG-PDLLAHMME, and PEG-PDLLA against HepG2 cells was measured, and the cellular reactive oxygen species, percentage of cells with mitochondrial membrane potential depolarization, and apoptotic rate following treatment were determined.

Results: Four nanovesicles (PEG-PDLLA-DOX-HMME, PEG-PDLLA-DOX, PEG-PDLLAHMME, and PEG-PDLLA) were synthesized, and mean particle sizes were $175 \pm 18 \mathrm{~nm}$, $154 \pm 3 \mathrm{~nm}, 196 \pm 2 \mathrm{~nm}$, and $147 \pm 15 \mathrm{~nm}$, respectively. PEG-PDLLA-DOX-HMME was more cytotoxic than PEG-PDLLA-DOX, PEG-PDLLA-HMME, and PEG-PDLLA. PEG-PDLLAHMME-treated cells had the highest mean fluorescence intensity, followed by PEG-PDLLADOX-HMME-treated cells, whereas PEG-PDLLA-DOX- and PEG-PDLLA-treated cells had a similar fluorescence intensity. Mitochondrial membrane potential depolarization was observed in $54.2 \%, 59.4 \%, 13.8 \%$, and $14.8 \%$ of the cells treated with PEG-PDLLA-DOX-HMME, PEGPDLLA-HMME, PEG-PDLLA-DOX, and PEG-PDLLA, respectively. The apoptotic rate was significantly higher in PEG-PDLLA-DOX-HMME-treated cells compared with PEG-PDLLADOX- and PEG-PDLLA-HMME-treated cells.

Conclusion: The PEG-PDLLA nanovesicle, a drug delivery carrier, can be simultaneously loaded with two anticancer drugs (hydrophilic DOX and hydrophobic HMME). PEG-PDLLADOX-HMME cytotoxicity to HepG2 cells is significantly higher than the PEG-PDLLA nanovesicle loaded with DOX or HMME alone, and DOX and HMME have a synergistic effect against human hepatocellular carcinoma HepG2 cells.

Keywords: polymeric vesicles, hematoporphyrin monomethyl ether, HMME, photodynamic therapy, PDT

\section{Introduction}

The incidence of hepatocellular carcinoma increases every year; however, the overall therapeutic efficacy and patients' survival rate remain unsatisfactory. ${ }^{1-3}$ Currently, most of the clinically available chemotherapeutic agents, such as doxorubicin (DOX), are small 
molecular compounds, which are problematic because they are not target specific, they induce multidrug resistance, and they have multiple side effects. ${ }^{4}$ Photodynamic therapy (PDT) is a form of phototherapy using light-sensitive compounds that are exposed selectively to light, which produces reactive oxygen species (ROS) and induces mitochondrial membrane potential depolarization to kill target tumor cells. ${ }^{5}$ PDT exhibits good specific targeting and low toxicity in tumor treatment, and causes minor damage to normal tissues; however, the poor water solubility of photosensitizers limits its wide application. ${ }^{5}$ Therefore, improving the targeting specificity of chemotherapeutic agents, reducing side effects, and increasing the water solubility of photosensitizers used in PDT is an effective strategy to enhance the therapeutic efficacy of antitumor drugs. These strategies have been given a high priority by physicians.

A polymeric nanovesicle is a nonviral carrier with a high biocompatibility, which achieves targeted drug delivery through the enhanced permeability and retention effect and through a connection with a targeted ligand. It is characterized by a long half-life, high stability, and minor side effects, which makes it suitable for the targeted delivery of antitumor agents. The polymeric nanovesicle is considered to have the potential to improve the unsatisfactory targeting of chemotherapeutic drugs and poor water solubility of photosensitizers, which has become a hot topic in nanomedicine research..$^{3,6-12}$
In the current study, a targeted nanovesicular drug delivery system with high-efficiency DOX and hematoporphyrin monomethyl ether (HMME) loading was studied. The polymeric nanovesicle poly(ethylene glycol)-block-poly (D,L-lactic acid) (PEG-PDLLA) was used as a carrier, and it was loaded with DOX and the photosensitizer HMME to form the nanovesicle PEG-PDLLA-DOX-HMME. The targeting specificity and cytotoxicity of PEG-PDLLA-DOX-HMME against human hepatocellular carcinoma HepG2 cells and its possible mechanism to induce apoptosis were investigated.

\section{Materials and methods Synthesis and characterization of mPEGIk-PDLLAIOk}

The synthesis of polyethylene glycol-polylactide (mPEG1kPDLLA10k) is shown in Figure 1A. Ethyl acetate was dried with calcium hydride and distilled for subsequent experiments. $\mathrm{D}$, L-lactide was recrystallized twice in anhydrous ethyl acetate to produce D,L-lactide crystal for the subsequent experiments. About 0.2 g monomethoxy polyethylene glycol (mPEG-OH; the number average molecular weight $(\mathrm{Mn})=1,000 \mathrm{~g} / \mathrm{mol}$ ) was added to a dry $100 \mathrm{~mL}$ reaction vessel, vacuum dried at $70^{\circ} \mathrm{C}$ for 4 hours until the absence of air bubbles, and cooled to room temperature. Then $0.5 \%$ stannous octanoate and $2.0 \mathrm{~g}$ D,L-lactide crystals were added under nitrogen protection,
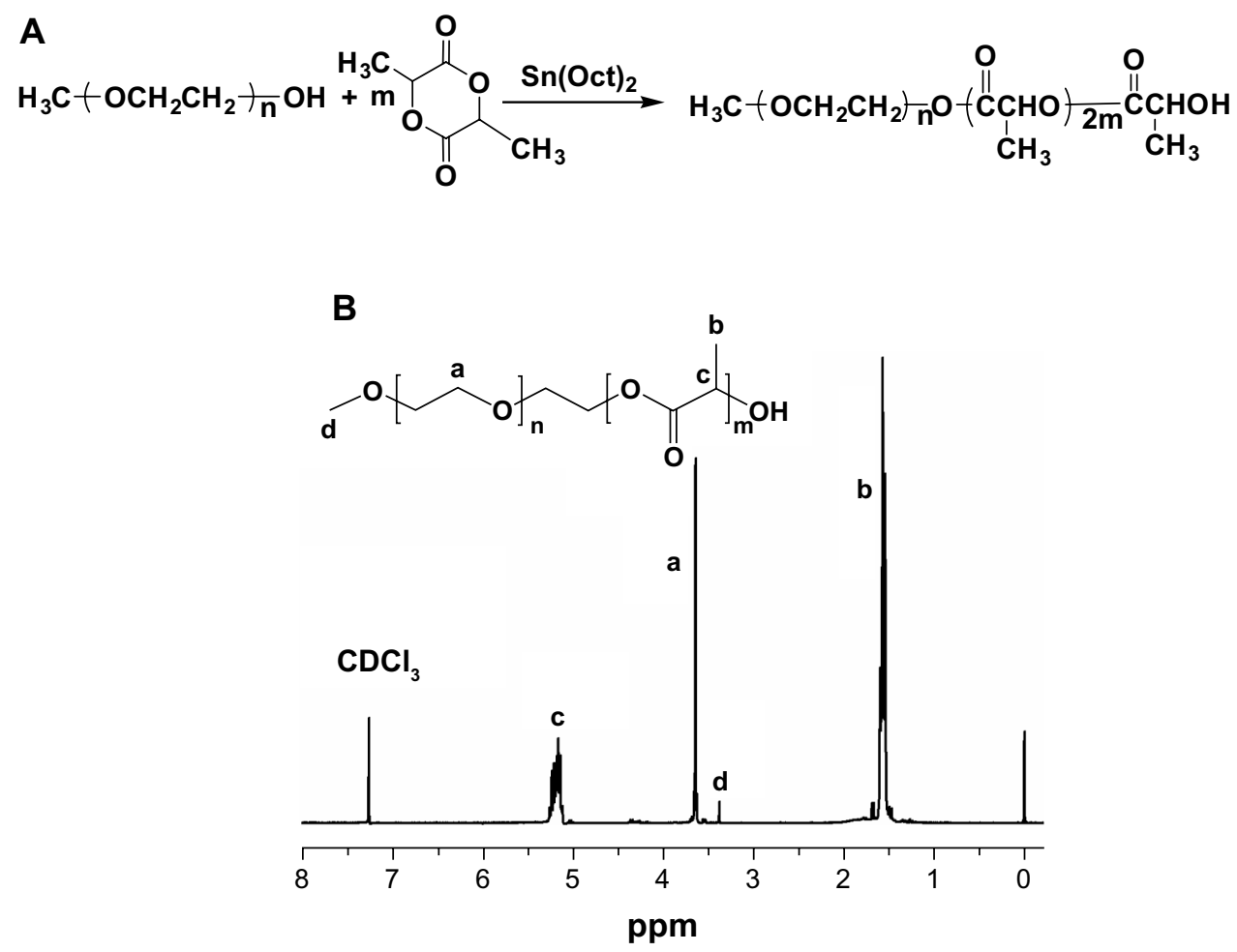

Figure I (A) The synthesis of poly(ethylene glycol)-block-poly(D,L-lactic acid) (PEGIk-PDLLAI0k) and (B) proton nuclear magnetic resonance spectra of mPEGIk-PDLLA in $\mathrm{CDCl}_{3}$ at $25^{\circ} \mathrm{C}$. Characteristic peaks of PEG and PDLLA were located at about $3.6 \mathrm{ppm}$ and $5.1-5.3 \mathrm{Ppm}(\mathrm{CH})$ and I.5-I.6 Ppm $\left(\mathrm{CH}_{3}\right)$, respectively. Abbreviation: Peglk-PDLLAI0k, polyethylene glycol-polylactide. 
vacuum dried at room temperature for 3 hours, and dissolved with $20 \mathrm{~mL}$ of newly distilled anhydrous toluene. The reflux reaction was performed at $120^{\circ} \mathrm{C}$ for 12 hours. The reaction system changed from a clear solution to white opacity. The reaction solution was transferred to absolute ether for reprecipitation, placed in a refrigerator overnight, leached, and dissolved with dichloromethane. The reaction solution was reprecipitated in absolute ether, leached, and dried, and the polymer samples were collected. The polymer structure was characterized using proton nuclear magnetic resonance spectroscopy (shown in Figure 1B) and infrared spectrum analysis.

\section{Synthesis of blank PEG-PDLLA nanovesicle}

The blank PEG-PDLLA nanovesicle was prepared using a double emulsion method. Briefly, $20 \mathrm{mg}$ PEG-PDLLA was dissolved in $2 \mathrm{~mL}$ of tetrahydrofuran, and $0.2 \mathrm{~mL}$ of distilled water was slowly added to the tetrahydrofuran solution in an ice bath. The solution underwent sonication to yield the initial emulsion solution, which was dropped into $20 \mathrm{~mL}$ of distilled water under the same conditions to yield the secondary emulsion solution. The secondary emulsion solution was incubated at room temperature to remove some tetrahydrofuran, and then dialysis was performed using a dialysis bag (molecular weight $=14 \mathrm{kDa}$ ) in water for 24 hours to remove the rest of the tetrahydrofuran. Large granules in the emulsion solution were removed using a $450 \mathrm{~nm}$ filter and stored at $4^{\circ} \mathrm{C}$ for the subsequent experiments.

\section{Synthesis of DOX-loaded PEG-PDLLA nanovesicles}

A total of $20 \mathrm{mg}$ PEG-PDLLA was dissolved in $2 \mathrm{~mL}$ of tetrahydrofuran. DOX aqueous solution $(0.2 \mathrm{~mL}$ contain $2 \mathrm{mg}$ DOX) was slowly dropped into the tetrahydrofuran solution under ice bath conditions and sonicated to yield the initial emulsion solution. This was dropped into $20 \mathrm{~mL}$ of distilled water under the same conditions to yield the secondary emulsion solution. Dialysis was performed using a dialysis bag (molecular weight $=14 \mathrm{kDa}$ ) in distilled water for 24 hours to remove organic solvents and DOX that was not encapsulated in the PEG-PDLLA nanovesicles. Large granules in the emulsion solution were removed using a $450 \mathrm{~nm}$ filter and stored at $4^{\circ} \mathrm{C}$ for the subsequent experiments.

\section{Synthesis of photosensitizer-loaded PEG-PDLLA nanovesicles}

Photosensitizer (2 mg) and $20 \mathrm{mg}$ PEG-PDLLA were simultaneously dissolved in $2 \mathrm{~mL}$ of tetrahydrofuran.
Distilled water $(0.2 \mathrm{~mL})$ was slowly dropped into the tetrahydrofuran solution under ice bath conditions and sonicated to yield the initial emulsion solution. This was dropped into $20 \mathrm{~mL}$ of distilled water under the same conditions to yield the secondary emulsion solution. Dialysis was performed using a dialysis bag (molecular weight $=14 \mathrm{kDa}$ ) in water for 24 hours to remove tetrahydrofuran. A $450 \mathrm{~nm}$ filter was used to remove the large granules and the photosensitizer that was not loaded in the hydrophobic layer of the nanovesicle, and stored at $4{ }^{\circ} \mathrm{C}$ for the subsequent experiments.

\section{Synthesis of PEG-PDLLA nanovesicles simultaneously loaded with DOX and photosensitizer}

Photosensitizer (2 mg) and $20 \mathrm{mg}$ PEG-PDLLA were simultaneously dissolved in $2 \mathrm{~mL}$ of tetrahydrofuran. Then DOX aqueous solution $(0.2 \mathrm{~mL}$ containing $2 \mathrm{mg}$ DOX) was slowly dropped into the tetrahydrofuran solution under ice bath conditions and sonicated to yield the initial emulsion solution, which was dropped into $20 \mathrm{~mL}$ of distilled water under the same conditions to yield the secondary emulsion solution. Subsequently, dialysis was performed using a dialysis bag (molecular weight $=14 \mathrm{kDa}$ ) in water for 24 hours to remove tetrahydrofuran and isolated DOX. A $450 \mathrm{~nm}$ filter was used to remove the large granules and the photosensitizer that was not loaded in the hydrophobic layer of the nanovesicle, and was stored at $4^{\circ} \mathrm{C}$ for the subsequent experiments.

\section{Characterization of the polymeric nanovesicles loaded with anticancer drugs}

The shape of the polymeric nanovesicle was observed using a transmission electron microscope, and the particle size and zeta potential of the nanovesicle were measured using dynamic laser scattering apparatus at $20^{\circ} \mathrm{C}$, with an incident laser wavelength $(\lambda)$ of $623 \mathrm{~nm}$ and an incident angle $(\theta)$ of $90^{\circ}$.

\section{DOX and HMME-loading content}

The DOX-loading content of DOX and HMME was quantified by ultraviolet-visible analysis using an ultravioletvisible near-infrared spectrophotometer (UV-3150; Shimadzu Corporation, Kyoto, Japan). The freeze-dried nanovesicles were weighed and redissolved in dimethyl sulfoxide before analysis. The absorbance of DOX at $482 \mathrm{~nm}^{13}$ and HMME at $539 \mathrm{~nm}^{14}$ were measured to determine the concentration in the solution using a pre-established calibration curve. 


\section{Cell culture and cytotoxicity detection}

Human hepatocellular carcinoma HepG2 cell lines were provided by the Laboratory Animal Center of Sun Yat-Sen University (Guangzhou, People's Republic of China) and passaged in Dulbecco's Modified Eagle's Medium at $37^{\circ} \mathrm{C}$ under saturated humidity containing $5 \% \mathrm{CO}_{2}$.

The cytotoxicity of four nanovesicles, PEG-PDLLADOX-HMME, PEG-PDLLA-DOX, PEG-PDLLA-HMME, and PEG-PDLLA, against HepG2 cells (at a density of $5 \times 10^{3}$ cells) was evaluated using the 3-[4,5-dimethylthiazol-2-yl]2,5-diphenyltetrazolium bromide assay, and the absorption value (A value) of each well at $570 \mathrm{~nm}$ was measured using a Wellscan Mk3 microplate reader (Labsystems, Dragon, Finland). All experiments were repeated in triplicate, and triple duplicate holes were used for each concentration. The survival rate of HepG2 cells was calculated using the following formula: cell survival rate $(\%)=A$ value in the experimental group/A value in the control group $\times 100 \%$.

\section{ROS detection in HepG2 cells treated with drug-loaded nanovesicles}

The ROS in HepG2 cells (at a density of $5 \times 10^{3}$ cells) treated with drug-loaded nanovesicles was detected using flow cytometry. 2', $7^{\prime}$-dichlorodihydrofluorescein diacetate (DCHF-DA), which enters cells to produce nonluminous DCHF, is a new-generation agent to capture ROS. If ROS is present in cells, oxidization occurs, resulting in production of fluorescence by the DCHF. Following endocytosis, PEG-PDLLA-HMME and PEG-PDLLA-HMME-DOX are released in cells, and the laser excitation oxidizes DCHF to DCHF-DA, which appears as green fluorescence. The intensity and quantity of green fluorescence can be used to reflect the ROS level.

\section{Detection of mitochondrial membrane potential depolarization in HepG2 cells treated with drug-loaded nanovesicles}

The mitochondrial membrane potential depolarization in HepG2 cells (at a density of $5 \times 10^{3}$ cells) treated with drugloaded nanovesicles was detected using flow cytometry. As an ideal probe to detect mitochondrial membrane potential depolarization, 5,5',6,6'-tetrachloro-1, 1',3,3'-tetraethylbenzimida zolcarbocyanine iodide (JC-1) is present as a monomer at a low mitochondrial membrane potential, which appears as red fluorescence (emission wavelength of 485/580 nm). JC-1 does not stay in apoptotic or necrotic cells, which appear as green fluorescence (emission wavelength of 485/530 nm), indicating mitochondrial membrane potential depolarization.
The ratio of green fluorescence intensity to salmon red fluorescence intensity can be used to reflect mitochondrial membrane potential depolarization. Following treatment with PEG-PDLLA-HMME, PEG-PDLLA-DOX, and PEG-PDLLA-HMME-DOX for 12 hours, the HepG2 cells were exposed to a laser at a certain wavelength $(632.8 \mathrm{~nm}$, $2.8 \mathrm{~J} / \mathrm{cm}^{2}, 30 \mathrm{~mW}$ ), and the ratio of green fluorescence intensity to salmon red fluorescence intensity reflects the change of mitochondrial membrane potential.

\section{Detection of apoptotic rate in HepG2 cells treated with drug-loaded nanovesicles}

The HepG2 cells (at a density of $5 \times 10^{3}$ cells) were cocultured with four nanovesicles for 12 hours, and the cells treated with PEG-PDLLA-HMME and PEG-PDLLA-HMME-DOX underwent PDT by exposure to a laser source $(632.8 \mathrm{~nm}, 2.8 \mathrm{~J} /$ $\mathrm{cm}^{2}, 30 \mathrm{~mW}$ ) at room temperature. HepG2 cell apoptosis was detected using an Annexin V-FITC Apoptosis Detection Kit (Keygen Biotech, Nanjing, People's Republic of China).

\section{Statistical analysis}

All statistical analyses were performed using the statistical software SPSS version 16.0 (SPSS Inc, Chicago, IL, USA). Differences in the proportion of HepG2 cells with mitochondrial membrane potential depolarization and HepG2 cell apoptotic rate were tested for statistical significance using an analysis of variance for multiple independent samples. Stepwise multiple comparisons were done using a StudentNewman-Keuls method. A $P$-value $<0.05$ was considered statistically significant.

\section{Results}

\section{Synthesis and characterization of polymeric nanovesicles}

Four polymeric nanovesicles were successfully synthesized, including PEG-PDLLA-DOX-HMME, PEG-PDLLADOX, PEG-PDLLA-HMME, and PEG-PDLLA (Figure 2). Transmission electron microscopy showed a clear structure of the synthesized nanovesicles, which appeared as hollow spheres with evenly distributed particle sizes (Figure 3). The mean PEG-PDLLA-DOX-HMME, PEG-PDLLA-DOX, PEG-PDLLA-HMME, and PEG-PDLLA particle sizes were $175 \pm 18 \mathrm{~nm}, 154 \pm 3 \mathrm{~nm}, 196 \pm 2 \mathrm{~nm}$, and $147 \pm 15 \mathrm{~nm}$, respectively, measured using a dynamic laser scattering apparatus (Figure 4). The mean blank nanovesicle PEG-PDLLA particle size was $147 \pm 15 \mathrm{~nm}$. The PEG-PDLLA nanovesicles had a narrow range of particle sizes, indicating that 

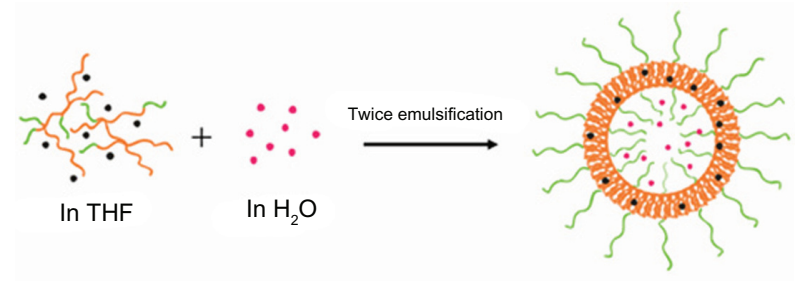

PEG حPDLLA . DOX . Photosensitizer

Figure 2 Synthesis of polymeric nanovesicles.

Abbreviations: DOX, doxorubicin; PDLLA, poly(D,L-lactic acid); PEG, poly(ethylene glycol); THF, tetrahydrofuran.

synthesized nanovesicles had homogeneous particle sizes. The mean PEG-PDLLA-DOX particle size was $154 \pm 3 \mathrm{~nm}$, and the particle sizes were distributed within a narrow range, demonstrating that the sizes of the nanovesicles did not change greatly and that they were distributed evenly following encapsulation of DOX. The mean particle size of the PEG-PDLLA nanovesicles loaded with photosensitizer alone was $196 \pm 2 \mathrm{~nm}$, which enlarged relative to the blank nanovesicle PEG-PDLLA. However, the particle size was still within the acceptable range, and was also distributed in a narrow range. The mean particle size of the nanovesicles loaded with DOX and photosensitizer simultaneously was smaller than that of the nanovesicles loaded with photosensitizer alone; however, the range of the particle sizes widened, indicating that the nanovesicles loaded with DOX and photosensitizer contained some blank nanovesicles or nanovesicles loaded with only one drug. The zeta potential of these nanovesicles ranges from $-1.67 \mathrm{mV}$ to $-0.65 \mathrm{mV}$, revealing a neutral surface.

The DOX concentration was $80 \mu \mathrm{g} / \mathrm{mL}$ and $60 \mu \mathrm{g} / \mathrm{mL}$ in the PEG-PDLLA-DOX-HMME and PEG-PDLLADOX nanovesicles, respectively, and the loading content was $4.4 \%$ and $3.4 \%$, respectively. The HMME concentration was $92 \mu \mathrm{g} / \mathrm{mL}$ and $130 \mu \mathrm{g} / \mathrm{mL}$ in the
PEG-PDLLA-DOX-HMME and PEG-PDLLA-HMME nanovesicles, respectively, and the loading content was $5.1 \%$ and $5.7 \%$, respectively.

\section{Cytotoxicity of synthesized polymeric nanovesicles}

The blank nanovesicle, PEG-PDLLA, showed a minor cytotoxicity to human hepatocellular carcinoma HepG2 cells, and there was no clear-cut concentration-dependent cytotoxicity observed (at concentrations of $1 \mu \mathrm{g} / \mathrm{mL}, 5 \mu \mathrm{g} / \mathrm{mL}, 10 \mu \mathrm{g} / \mathrm{mL}$, $15 \mu \mathrm{g} / \mathrm{mL}, 20 \mu \mathrm{g} / \mathrm{mL}, 25 \mu \mathrm{g} / \mathrm{mL}, 50 \mu \mathrm{g} / \mathrm{mL}$, and $100 \mu \mathrm{g} / \mathrm{mL}$ ) (Figure 5).

Following treatment with PEG-PDLLA-DOX and PEG-PDLLA-HMME for 24 hours, the survival rate of HepG2 cells gradually reduced with the increase in the nanovesicle concentration. Treatment with approximately $40 \%$ of the HepG2 cells survived the treatment with PEGPDLLA-DOX at a concentration of $4 \mu \mathrm{g} / \mathrm{mL}$ for 24 hours, whereas less than $10 \%$ of the cells survived the treatment with PEG-PDLLA-HMME using the same protocol. PEGPDLLA-DOX-HMME treatment caused a significantly lower survival of the HepG2 cells relative to PEG-PDLLA-DOX and PEG-PDLLA-HMME, and the cell mortality caused by PEG-PDLLA-DOX-HMME treatment was similar to the sum of the cell mortality caused by PEG-PDLLA-DOX and PEG-PDLLA-HMME treatments (Figure 6).

\section{ROS levels in HepG2 cells following treatment with drug-loaded nanovesicles}

The highest mean fluorescence intensity $(7,294)$ was detected in PEG-PDLLA-HMME-treated HepG2 cells, followed by PEG-PDLLA-DOX-HMME-treated cells $(3,149)$, whereas similar mean fluorescence intensity was observed in PEG-PDLLA-DOX- $(1,480)$ and PEG-PDLLA-treated HepG2 cells $(1,268)$ (Figure 7).

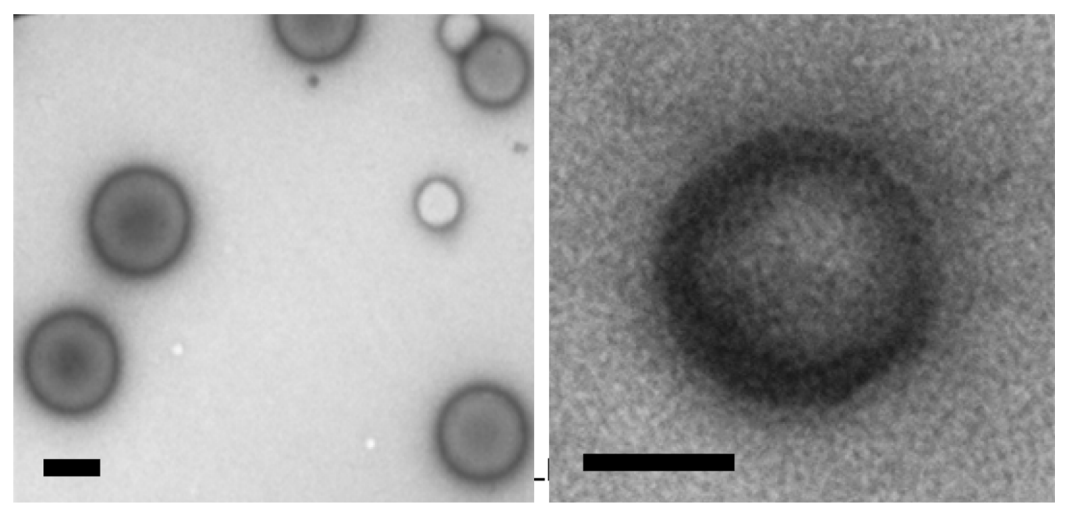

Figure 3 Transmission electron microscope photos of poly(ethylene glycol)-block-poly(D,L-lactic acid) vesicles. The structure of synthesized nanovesicles was clear, which was hollow and spherical. Particle size distribution was relatively homogeneous. 

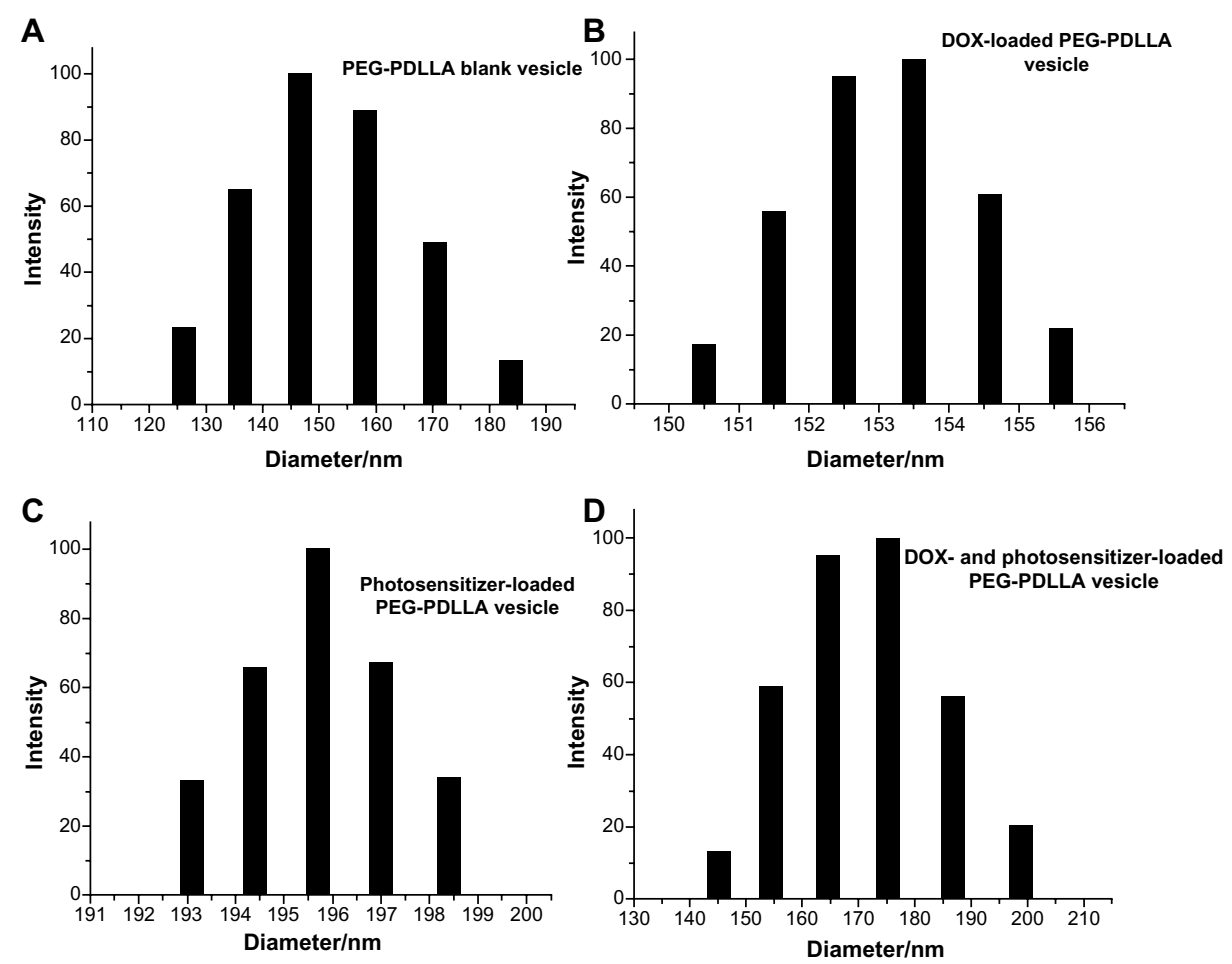

Figure 4 Particle sizing distribution of (A) poly(ethylene glycol)-block-poly(D,L-lactic acid) (PEG-PDLLA) blank vesicle, (B) doxorubicin (DOX)-loaded PEG-PDLLA vesicle, (C) photosensitizer-loaded PEG-PDLLA vesicle, and (D) DOX- and photosensitizer-loaded PEG-PDLLA vesicle.

\section{Mitochondrial membrane potential} depolarization in HepG2 cells following treatment with drug-loaded nanovesicles Treatment with PEG-PDLLA-DOX-HMME, PEGPDLLA-HMME, PEG-PDLLA-DOX, and PEG-PDLLA caused mitochondrial membrane potential depolarization in $54.2 \%, 59.4 \%, 13.8 \%$, and $17.7 \%$ of the HepG2 cells, respectively (Figure 8). The proportion of the HepG2 cells with mitochondrial membrane potential depolarization following PEG-PDLLA treatment was significantly higher than that of the cells treated with PEG-PDLLA-DOXHMME and PEG-PDLLA-HMME ( $F=7.783$ and 6.843, both $P$-values $<0.001$ ); however, there was no significant difference observed between the PEG-PDLLA-DOX

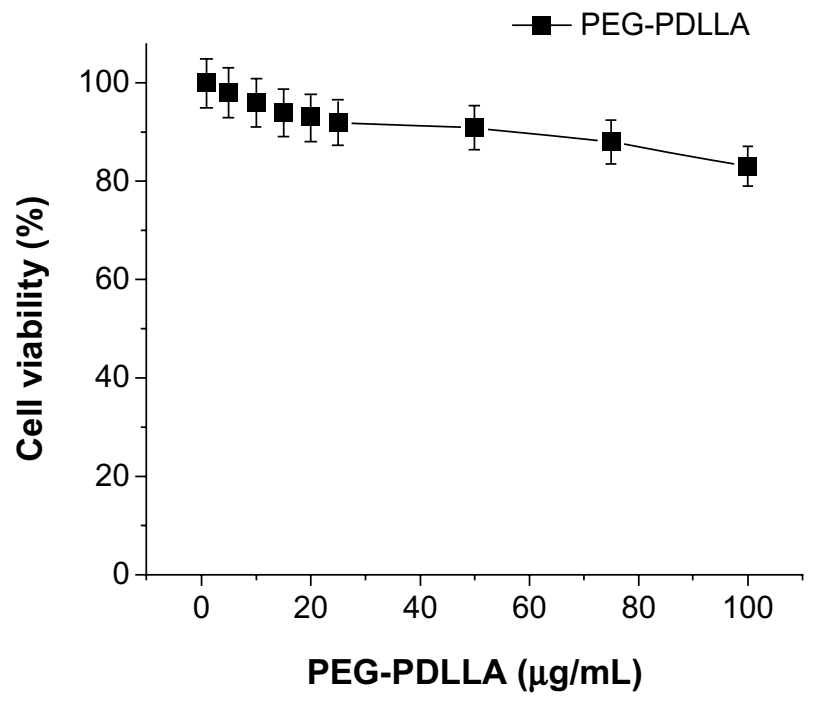

Figure 5 The toxicity of empty nanovesicles poly(ethylene glycol)-block-poly(D,Llactic acid) (PEG-PDLLA) on HepG2.

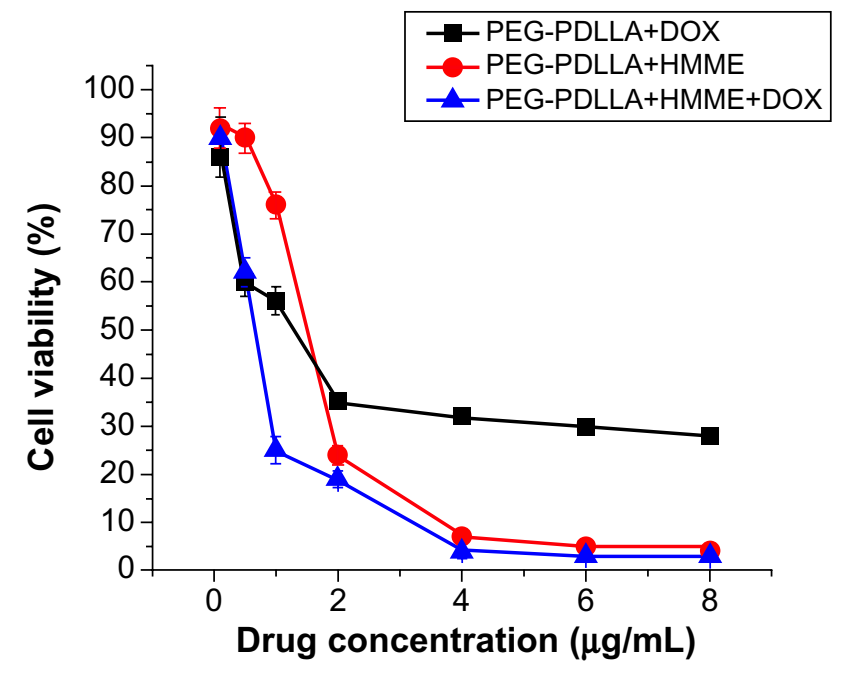

Figure $\mathbf{6}$ The toxicity of three groups of drug-loading nanoparticle vesicles. Abbreviations: DOX, doxorubicin; HMME, hematoporphyrin monomethyl ether; PDLLA, poly(D,L-lactic acid); PEG, poly(ethylene glycol). 


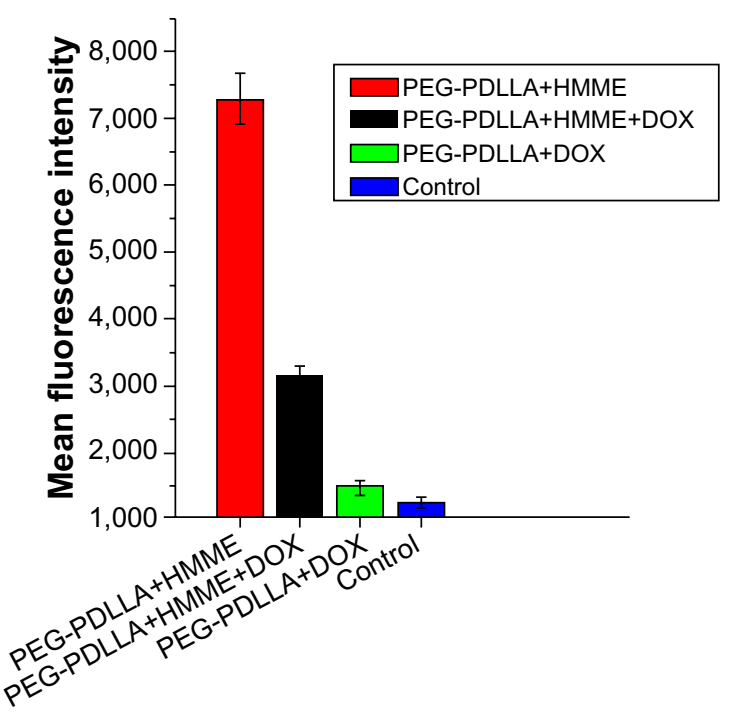

Figure 7 Reactive oxygen species levels in HepG2 cells following treatment with drug-loaded nanovesicles.

Abbreviations: DOX, doxorubicin; HMME, hematoporphyrin monomethyl ether; PDLLA, poly(D,L-lactic acid); PEG, poly(ethylene glycol).

treatment group and the PEG-PDLLA treatment group $(P>0.05)$.

\section{Apoptotic rate of HepG2 cells following treatment with drug-loaded nanovesicles}

Flow cytometry showed a significantly higher apoptotic rate in PEG-PDLLA-DOX-HMME-treated HepG2 cells
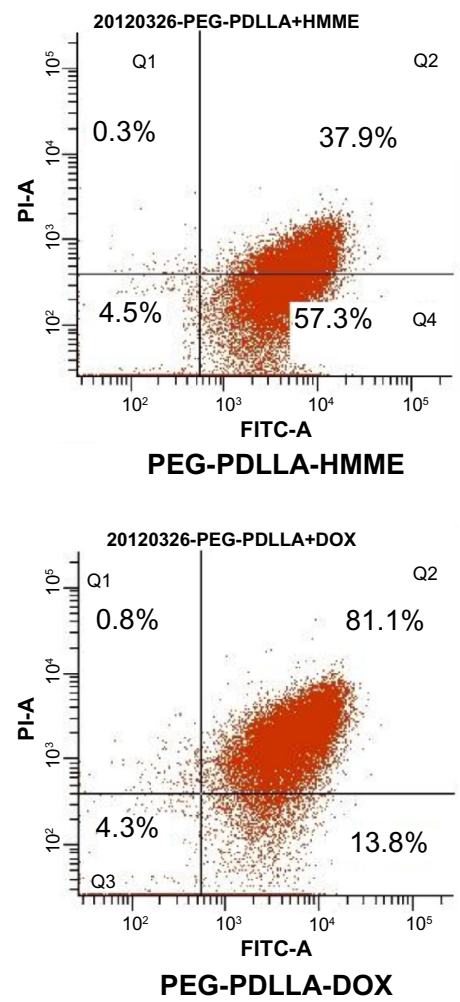

$(41.2 \%)$ than in the PEG-PDLLA-DOX-treated cells (17.2\%) and PEG-PDLLA-HMME-treated cells (14.5\%) (Figure 9). Following treatment with PEG-PDLLA-DOX and PEG-PDLLA-HMME for 12 hours, more HepG2 cells underwent apoptosis at an early stage compared with those that underwent apoptosis at an advanced stage and necrosis. The number of apoptotic cells in the PEG-PDLLA-DOXHMME treatment group was the sum of the apoptotic cells in the PEG-PDLLA-DOX and PEG-PDLLA-HMME treatment groups, which were mainly characterized by advanced-stage apoptosis and necrosis. The apoptotic rate of PEG-PDLLADOX-HMME-treated HepG2 cells was significantly higher than that of PEG-PDLLA-DOX-treated cells and PEGPDLLA-HMME-treated cells $(F=3.137$ and 2.594 , both $P$-values $<0.001)$.

\section{Discussion}

Hepatocellular carcinoma is characterized by a high incidence, high malignancy, poor prognosis, and relatively occult onset. Some patients who are at a moderate or advanced stage at diagnosis miss the optimal timing for surgery. Many chemotherapeutic drugs have problems such as short half-life and a large number of side effects, resulting in poor therapeutic efficacy. DOX is a common chemotherapeutic agent that is currently used in cancer therapy regimens, and it has a wide
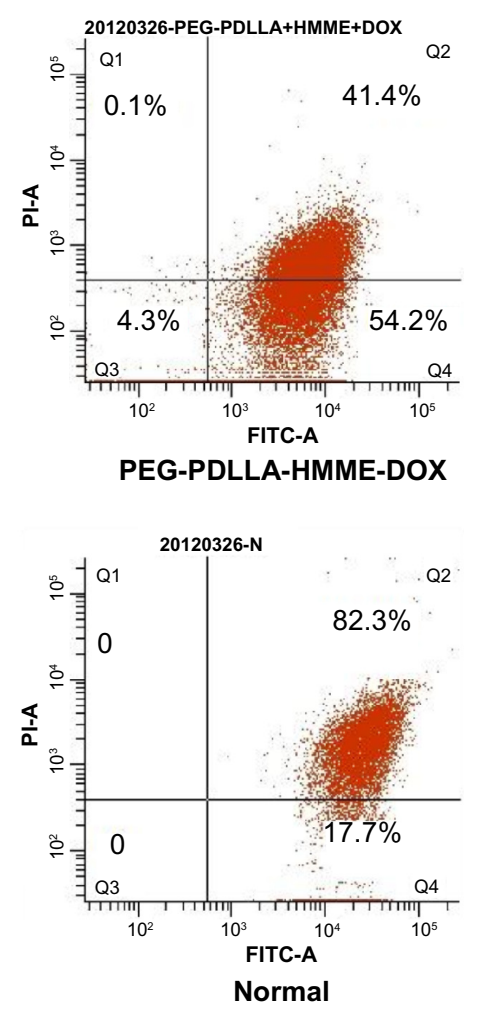

Figure 8 Mitochondrial membrane potential depolarization in HepG2 cells following treatment with drug-loaded nanovesicles.

Abbreviations: DOX, doxorubicin; HMME, hematoporphyrin monomethyl ether; PDLLA, poly(D,L-lactic acid); PEG, poly(ethylene glycol); FITC-A, fluorescein isothiocyanate apoptosis; PI-A, propidine iodide apoptosis; Q, quadrant. 


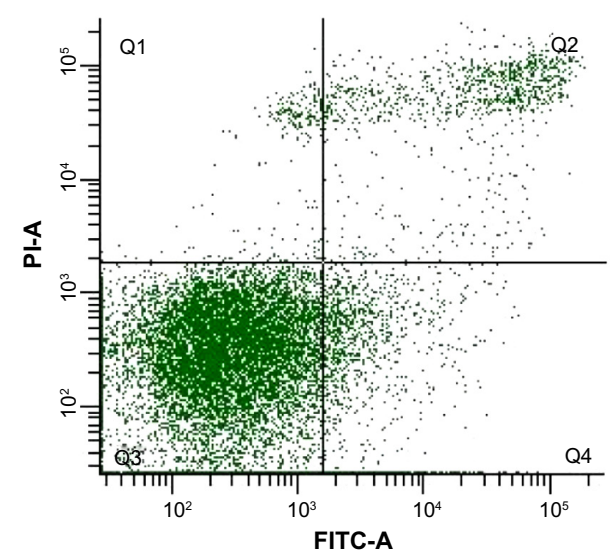

PEG-PDLLA-HMME

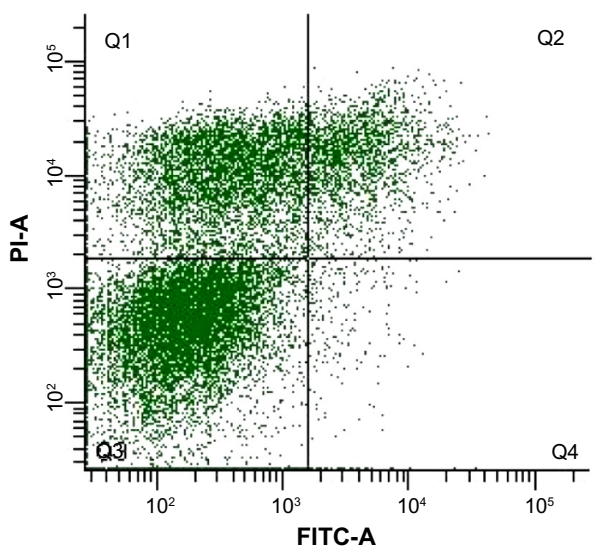

PEG-PDLLA-HMME-DOX
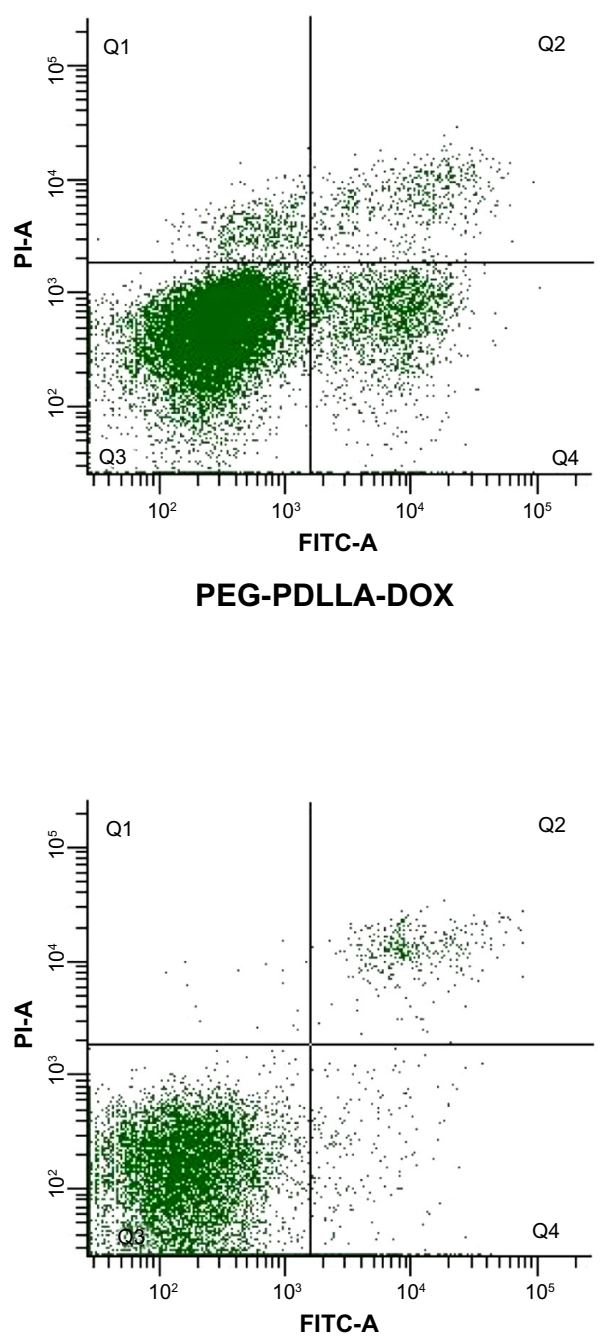

Control

Figure 9 The apoptosis-inducing rate test result of drug-loaded nanovesicles.

Abbreviations: DOX, doxorubicin; HMME, hematoporphyrin monomethyl ether; PDLLA, poly(D,L-lactic acid); PEG, poly(ethylene glycol); FITC-A, fluorescein isothiocyanate apoptosis; Pl-A, propidine iodide apoptosis; Q, quadrant.

anticancer spectrum. However, DOX has problems such as high toxicity to normal tissues, inevitable cardiac toxicity, and myelosuppression. ${ }^{15}$ In addition, DOX stimulates the production of multiple antiapoptotic proteins such as Bcl-2, Bcl-xl, and multidrug-resistant proteins in tumor cells that avoid the DOX effect, resulting in the development of multidrug resistance. The chemotherapeutic agents have a killing effect on tumor cells and also result in much damage to normal human cells such as hematopoietic cells and gastrointestinal mucosal cells; this results in severe side effects that cause some patients to refuse or abandon the treatment.

PDT was approved by the US Food and Drug Administration for clinical use in 1996. The ROS generated by the photodynamic reaction is found to directly kill cells; in addition, it has been recently shown that PDT can activate the immune response against tumors and destroy the blood vessels in tumor tissues to block the blood supply to tumors. Photosensitizers or their metabolites are compounds that can selectively gather at the target site, and the ROS generated by exposure to light at a certain wavelength can kill the cells in the tissues surrounding the target site, causing cytotoxicity. ${ }^{16}$ The photosensitizer plays a decisive role in promoting the clinical application of PDT. HMME is a photosensitizer that is selectively absorbed by tumor tissues, and it has a high photosensitive effect, a low toxicity, and a chemical purity of $99.8 \%$. HMME is rapidly excreted, and it exhibits a similar in vitro photosensitive killing efficiency against tumor cells as do the second-generation photosensitizers aminolevulinic acid and benzoporphyrin-derivative monoacid ring A. ${ }^{17}$ However, the problem of comparatively poor water solubility limits the wide application of photosensitizers in clinical practice. 
The search for an effective targeted drug delivery system with the aim to improve chemotherapeutic agent targeting and solubility of photosensitizers in water has become the subject of much tumor chemotherapy research. Polymeric nanovesicles are multifunctional composite nanoparticles. Our previous studies showed that the polymeric nanovesicles have several specific advantages, which include their small size, long half-life, and the ability to easily achieve both passive and active targeted delivery; these advantages make nanovesicles an excellent carrier that is suitable for the targeted delivery of hydrophobic drugs. ${ }^{6-9}$ The nanovesicle has a specific nuclear shell structure with a hydrophilic surface and a hydrophobic inner structure. The hydrophilic shell allows the nanoparticle to remain stable in a liquid environment, and it is also readily dispersed in aqueous solutions, which allows it to be transferred to specific sites via the blood circulation. In addition, the biologically, physically, and chemically modified shell has a targeting function. The inner nucleus of the nanovesicle may serve as a nanodrug store for the controlled release of hydrophobic drugs, and it can also carry metal compounds and large molecules with charges such as proteins and nucleotides. The specific size $(10-150 \mathrm{~nm})$ of the nanovesicle is beneficial because it reduces the duration of renal excretion and clearance, reticuloendothelial system uptake, and phagocytic recognition, thereby prolonging the circulation time. In addition, the nanovesicle can reach specific areas such as tumors via the vascular endothelial gap through the enhanced permeability and retention effect, in order to passively target the tumor. The nanovesicle can be used as a carrier to achieve targeted drug delivery and controlled release, which reduces drug contact with normal tissues and cells and is beneficial for the reduction of drug side effects. It is considered that the biodegradable nanovesicle has many advantages in drug delivery, including stable characteristics and a high degree of biocompatibility, which suggests wide application prospects in drug delivery.

\section{Conclusion}

In the present study, the polymeric nanovesicle was used as a drug carrier, and it was simultaneously loaded with DOX and HMME. We aimed to address the unsatisfactory DOX targeting, a large number of side effects, and poor water solubility of the photosensitizer, and thereby develop a novel multifunctional targeted nanovesicular drug delivery system. Our preliminary findings demonstrate that the nanovesicle PEG-PDLLA has minor side effects, and that it can be efficiently loaded with two anticancer agents simultaneously.
In addition, the PEG-PDLLA nanovesicle loaded with DOX and HMME was found to exhibit better efficacy against human hepatocellular carcinoma HepG2 cells than the PEGPDLLA nanovesicle loaded with DOX or HMME alone.

In conclusion, the PEG-PDLLA nanovesicles, when used as drug delivery carriers, have the potential to help reduce side effects of DOX and improve HMME water solubility. The PEG-PDLLA nanovesicles also allow a synergistic effect of DOX and HMME on human hepatocellular carcinoma cells, which requires further study.

\section{Acknowledgments}

This research was supported by the National Natural Science Foundation of China (81271561, 30900357, $51373203,21174166)$ and the Natural Science Foundation of Guangdong Province (S2012010008942, 9451008901001949 , S2012020011070), and SYSU project for young teacher (11LGPY44, 10YKPY08).

\section{Disclosure}

The authors report no conflicts of interest with this work.

\section{References}

1. World Health Organization. Cancer. Available from: http://www.who. int/mediacentre/factsheets/fs297/en/index.html. Accessed October 30, 2013.

2. Hong GB, Zhou JX, Shen J, et al. Preparation of a folate-mediated tumor targeting ultraparamagnetic polymeric micelles and its in vitro experimental study. Chin J Radiol. 2008;42(1):19-23.

3. Cheng D, Hong GB, Wang WW, et al. Nonclustered magnetite nanoparticle encapsulated biodegradable polymeric micelles with enhanced properties for in vivo tumor imaging. J Mater Chem. 2011;21(13): 4796-4804.

4. Gu YJ, Cheng J, Jin J, et al. Development and evaluation of pHresponsive single-walled carbon nanotube-doxorubicin complexes in cancer cells. Int J Nanomedicine. 2011;6:2889-2898.

5. Mitton D, Ackroyd R. A brief overview of photodynamic therapy in Europe. Photodiagnosis Photodyn Ther. 2008;5(2):103-111.

6. Shuai X, Ai H, Nasongkla N, et al. Micellar carriers based on block copolymers of poly(epsilon-caprolactone) and poly(ethylene glycol) for doxorubicin delivery. J Control Release. 2004;98(3): 415-426.

7. Niu CQ, Sun QQ, Zhou JX, et al. Folate-functionalized polymeric micelles based on biodegradable PEG-PDLLA as a hepatic carcinomatargeted delivery system. Asian Pac J Cancer Prev. 2011;12(8): 2071-2075

8. Hong G, Yuan R, Liang B, et al. Folate-functionalized polymeric micelle as hepatic carcinoma-targeted, MRI-ultrasensitive delivery system of antitumor drugs. Biomed Microdevices. 2008;10(5):693-700.

9. Wang W, Cheng D, Gong F, et al. Design of multifunctional micelle for tumor-targeted intracellular drug release and fluorescent imaging. Adv Mater. 2012;24(1):115-120.

10. Yang XQ, Deng WJ, Fu LW, et al. Folate-functionalized polymeric micelles for tumor targeted delivery of a potent multidrug-resistance modulator FG020326. J Biomed Mater Res A. 2008;86(1):48-60.

11. Nasongkla N, Bey E, Ren J, et al. Multifunctional polymeric micelles as cancer-targeted, MRI-ultrasensitive drug delivery systems. Nano Lett. 2006;6(11):2427-2430. 
12. Ai H, Flask C, Weinberg B, et al. Magnetite-loaded polymeric micelles as ultrasensitive magnetic-resonance probes. Adv Mater. 2005;17(16): 1949-1952.

13. Dai J, Lin S, Cheng D, et al. Interlayer-crosslinked micelle with partially hydrated core showing reduction and $\mathrm{pH}$ dual sensitivity for pinpointed intracellular drug release. Angew Chem Int Ed Engl. 2011;50(40): 9404-9408.

14. Lei TC, Glazner GF, Duffy M, et al. Optical properties of hematoporphyrin monomethyl ether (HMME), a PDT photosensitizer. Photodiagn Photodyn. 2012;9(3):232-242.
15. Parkin DM, Bray F, Ferlay J, et al. Estimating the world cancer burden: Globocan 2000. Int J Cancer. 2001;94(2):153-156.

16. Sharman WM, Allen CM, van Lier JE. Photodynamic therapeutics: basic principles and clinical applications. Drug Discov Today. 1999;4(11): 507-517.

17. Huang X, Zhou S. Photodynamic inhibition of hematoporphyrin monomethyl ether in hepatoma cells. Laser J. 2010;31(4):76-77.

\section{Publish your work in this journal}

The International Journal of Nanomedicine is an international, peerreviewed journal focusing on the application of nanotechnology in diagnostics, therapeutics, and drug delivery systems throughout the biomedical field. This journal is indexed on PubMed Central, MedLine, CAS, SciSearch $\AA$, Current Contents ${ }^{\circledR} /$ Clinical Medicine,
Journal Citation Reports/Science Edition, EMBase, Scopus and the Elsevier Bibliographic databases. The manuscript management system is completely online and includes a very quick and fair peer-review system, which is all easy to use. Visit http://www.dovepress.com/ testimonials.php to read real quotes from published authors. 